\title{
Hepatology training in the UK
}

Imran Patanwala, ${ }^{1,2}$ Mark Hudson ${ }^{1-3}$

'Liver Unit Freeman Hospital, Newcastle upon Tyne, UK ${ }^{2}$ Institute of Cellular Medicine, Newcastle University, Newcastle upon Tyne, UK

${ }^{3}$ British Association for the Study of the Liver, London, UK

\section{Correspondence to}

Dr Mark Hudson, Liver Unit, Freeman Hospital, Newcastle upon Tyne NE7 7DN, UK; Mark.hudson@nuth.nhs.uk

Accepted 12 October 2011 Published Online First

11 November 2011

\begin{abstract}
Objectives To establish the perceived adequacy of the hepatology training component of the unified gastroenterology and hepatology training programme in the UK by assessing the attitudes and experiences of trainees in the programme.
\end{abstract}

Design and intervention Online cross-

sectional questionnaire survey linked to the annual British Society of Gastroenterology/ Trainee in Gastroenterology survey in 2010.

Setting and participants National survey of all specialist gastroenterology trainees in the UK. Results 283/489 (58\%) trainees responded, 68\% were male. $54 \%$ of all trainees wanted to deliver liver services as consultants. $25 \%$ of trainees complete training without exposure to a liver unit providing comprehensive specialist hepatology services. Median time spent in such a unit for the others was 8 months (IQR 6). Significantly fewer trainees lacked confidence in managing liverrelated conditions if they had spent time training in a specialist liver unit and with increasing years in training. One in three trainees is dissuaded from a career in hepatology. One in five trainees wished to work part time as consultants-an option preferred significantly more by women. Conclusions Hepatology training in the UK is perceived by trainees as being suboptimal. A national strategy aimed at improving and standardising hepatology training and making specialist liver unit experience available for every trainee is required.

\section{Introduction}

Liver disease is the fifth most common cause of death in the UK and the burden of liver disease in the UK is rising. Hospital admission rates for liver disease between 1990 and 2003 increased by $70 \%$ for men and $40 \%$ for women. ${ }^{1}$ National standardised mortalities from liver disease for men increased by $104 \%$ in 2005 compared with those in $1991 .^{2}$ There has been a sixfold increase in liver disease and liver deaths in the past 35 years. Establishment of two additional liver transplant centres 20 years ago, modifications in the gastroenterology training curriculum and recognition of hepatology as a subspecialty by the Royal College of Physicians in 2005 have attempted to keep up with this increase in liver-related morbidity and mortality, increasing both the scope and opportunity for hepatology training in the country. However, a recent white paper by British Association for Studies of the Liver (BASL) and the British Society of Gastroenenterology (BSG) expressed concern that there has been little change in the organisation of liver services or hepatology training in the $\mathrm{UK}^{3}$

The majority of secondary liver care is provided by gastroenterologists working in district general hospital (DGHs). In a recent survey, liver disease contributed to approximately $20 \%$ of their workload. ${ }^{4}$ However, only one DGH had a full-time dedicated hepatologist, only $8 \%$ of the DGHs had a gastroenterologist with a special interest in hepatology and almost half $(44 \%)$ of the gastroenterologists had not received formal training in hepatology. ${ }^{4}$ The provision of liver services in the UK is suboptimal. ${ }^{34}$ To overcome this, a national strategy for liver services in the UK is being formulated for the Department of Health by the national clinical director for liver services as a result of the jointly produced paper by BASL and BSG. ${ }^{3}$ This envisages a consultant trained in advanced hepatology in every DGH by 2016 .

Training in hepatology in the UK is offered through unified, geographically defined, combined gastroenterology and hepatology training programmes to specialist trainees. Specialist training in general internal medicine is also offered in these programmes throughout the UK. One-year hepatology fellowships for those desiring specialist accreditation in hepatology were created in 2004. However, they cater for only a small fraction (16 trainees) of the national trainee pool who are eligible (specialist training year 3 and above) to apply for these posts each year. At present, some of these specialist posts remain empty. Training in hepatology for a large proportion of UK gastroenterology 
Table 1 Preferred goal consultant jobs after completion of training

\begin{tabular}{ll}
\hline Category & Description of category \\
\hline 1 & $\begin{array}{l}\text { A gastroenterologist with no interest in looking after patients } \\
\text { with liver disease except when they are acutely admitted on } \\
\text { my take }\end{array}$ \\
& $\begin{array}{l}\text { A gastroenterologist with a specific interest in hepatology } \\
\text { providing specialist care for patients with liver disease } \\
\text { (including specialist liver clinics) but relying on a regional } \\
\text { centre for more advice and shared care }\end{array}$ \\
3 & $\begin{array}{l}\text { A hepatologist working in a transplant or non-transplant } \\
\text { regional centre providing comprehensive hepatology } \\
\text { services, including specialist liver referral clinics and providing } \\
\text { specialised advice to a secondary care centre } \\
\text { An academic hepatologist }\end{array}$ \\
\hline
\end{tabular}

trainees is, therefore, embedded in the local rotational programme, which varies between regions according to the specialist liver experience that is on offer. To deliver the recommendations of the National Plan for Liver Services, data are needed on the proportion of trainees interested in delivering specific liver services and the adequacy of opportunities available to them within current gastroenterology training rotations. Finally, current trainees' views on obstacles, perceived or real, which would dissuade them from taking up a career in hepatology need to be identified. This is particularly pertinent and may explain why some advanced hepatology training posts lie empty each year. The aims of this survey were to collect such data from the gastroenterology and hepatology trainee pools in the UK.

\section{Methods}

Cross-sectional data were collected using an online survey, which was emailed to 489 trainees in gastroenterology in the UK in April 2010 incorporated into the BSG/Trainees in Gastroenterology annual survey. This is sent to every specialist gastroenterology trainee in the UK. The number of trainees in the UK is greater than 489 , but we could only ascertain that 489 received this survey. Questions included in the questionnaire were reviewed by training and hepatology leads and piloted among local trainees. A financial reward sponsored by the BASL was announced for one random respondent to improve response rates. To identify future preferred work patterns in the provision of hepatology services, we created four categories as shown in table 1.

A unit providing comprehensive liver services was defined as one which supplies treatment of viral hepatitis, management of hepatocellular carcinoma and $24 \mathrm{~h}$ services for emergency management of variceal bleeding, including specialised endoscopic and radiological interventions, with or without transplanting facilities. All the data were collected and analysed using Excel 2007 and statistics were performed using Minitab 15. Differences in proportions were tested using Fisher's exact test or $\chi^{2}$ test. This survey did not need ethical approval and consent was implied when completed questionnaires were returned.

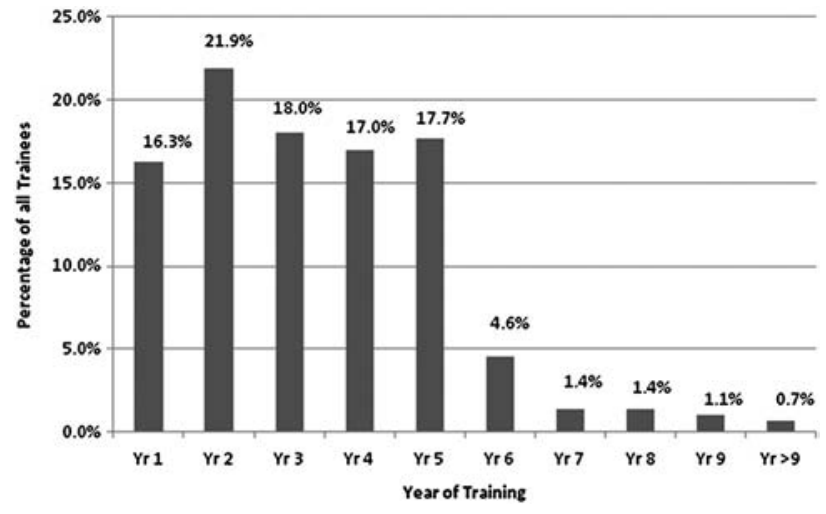

Figure 1 Proportion of trainees in each year of training.

\section{Results}

Response rates and demographics

Two hundred and eighty-three of 489 (58\%) trainees from 136 hospitals in 20 deaneries across England, Wales, Scotland and Northern Ireland responded to the survey. Sixty-eight per cent were male. Trainees from each year of training from year 1 to 5 were represented in equal proportions $\left(\chi^{2}=3.02, \mathrm{p}=0.555\right)$, year 5 being the final year of training for the majority of trainees (figure 1). The proportion of trainees in various training grades responding is given in table 2.

A quarter $(24 \%)$ of trainees were on an out of programme experience (OOPE), 74\% working towards the award of a postgraduate research degree; $20 \%$ had undergone an OOPE, again mainly for research.

\section{Preferred consultant work pattern and interest in hepatology}

Forty-six per cent (95\% CI $40 \%$ to $52 \%)$ of all trainees wish to work in category 1 consultant job profiles, $36 \%$ (95\% CI $30 \%$ to $42 \%$ ) in category 2, $14 \%$ (95\% CI $10 \%$ to $19 \%$ ) in category 3 and $4 \%$ (95\% CI $2 \%$ to $8 \%$ ) in a category 4 consultant job profile after their training (figure 2). Over half of all trainees $(54 \%, 95 \%$ CI $48 \%$ to $60 \%$ ), therefore wished to deliver liver services as consultants. There was no significant difference in the ratio of men to women opting for each category in comparison with the whole trainee pool $(\mathrm{p}=0.3)$. One in five trainees wish to work part time as consultants. Female trainees preferred future part-time consultant posts significantly more than their male counterparts ( $42 \%$ vs $8 \%, p<0.001$, Fisher's exact test).

\section{Current hepatology training opportunities}

Half of all trainees $(54 \%, 95 \%$ CI $47 \%$ to $60 \%$ ) and a quarter of trainees in year 5 or more of training $(25 \%, 95 \%$ CI $15 \%$ to $37 \%)$ stated that they had not yet received training in a specialist liver unit that provides comprehensive hepatology services as previously defined. A fifth of all trainees (19\%, 95\% CI $13 \%$ to $26 \%$ ) stated that it was unlikely that they would have such experience during their programmed regional rotation. Of those trainees who had not yet 
Table 2 Demographics and description of trainees who responded

\begin{tabular}{ll}
\hline Cohort description and demographics & \\
\hline Response rates, $\mathrm{n}(\%)$ & $283 / 489(58)$ \\
Male/female, $\mathrm{n}(\%)$ & $193 / 90(68: 32)$ \\
Specialist registrars, $\mathrm{n}(\%)$ & $150(53)$ \\
Specialist trainees, $\mathrm{n}(\%)$ & $107(38)$ \\
Locums approved for training, $\mathrm{n}(\%)$ & $12(4)$ \\
Academic clinical fellows, $\mathrm{n}(\%)$ & $4(1)$ \\
Others, $\mathrm{n}(\%)$ & $10(4)$ \\
Previous out of programme experience (OOPE), \% & 19 \\
Currently on OOPE, \% & 24 \\
Trained in advanced hepatology or intended to train in & $89(31)$ \\
advanced hepatology, $\mathrm{n}(\%)$ & \\
Pursuing academic hepatology or intending to pursue & $32(11)$ \\
academic hepatology, $\mathrm{n}(\%)$ & \\
\hline
\end{tabular}

been placed in such a unit but felt that such placement would occur, one-third $(30 \%, 95 \%$ CI 23\% to 39\%) thought this was most likely but did not know when and for how long, one-third (28\%, 95\% CI 21\% to $37 \%)$ thought that this would be a part of their rotation but for $<6$ months, one-fifth $(19 \%$, CI $13 \%$ to $26 \%$ ) thought that this would be a part of their rotation and would be for 6-12 months and 4\% (95\% CI 1\% to $8 \%$ ) stated that this would be part of their planned hepatology subspecialty CCST training. The median time spent by those who had worked in a hepatology unit was 8 months (IQR 6). One-third of trainees do not think that there are adequate training opportunities in their regional rotations to achieve competence in advanced hepatology or academic hepatology $(31 \%$, $95 \%$ CI $26 \%$ to $37 \%$ and $28 \% 95 \%$ CI $22 \%$ to $33 \%$, respectively).

\section{Obstacles to training in hepatology}

One in three of all trainees (32\%, 95\% CI 26\% to 40\%) and $41 \%(95 \%$ CI $32 \%$ to $51 \%)$ of those trainees wishing to deliver liver services as a consultant felt that there were obstacles that would dissuade them from pursuing a career in hepatology. Poor hepatology and liver transplant training, a lack of hepatology training numbers and importantly, a perceived lack of consultant-level jobs in hepatology, the need to relocate to take part in such training and being excluded from colonoscopy training (thus potentially reducing job opportunities) were cited as the five main reasons (figure 3 ).

\section{Adequacy of training as perceived by trainees}

One in five of all trainees $(20 \%, 95 \%$ CI $15 \%$ to $26 \%$ ) and a similar proportion of trainees in year 5 or higher $(15 \%, 95 \%$ CI $7 \%$ to $25 \%)$ stated that the hepatology training they will receive during their programmed rotation will be inadequate to help them confidently manage hepatological problems as a consultant. A large majority $(85 \%, 95 \%$ CI $79 \%$ to $89 \%)$ felt that their current training programme could be improved.

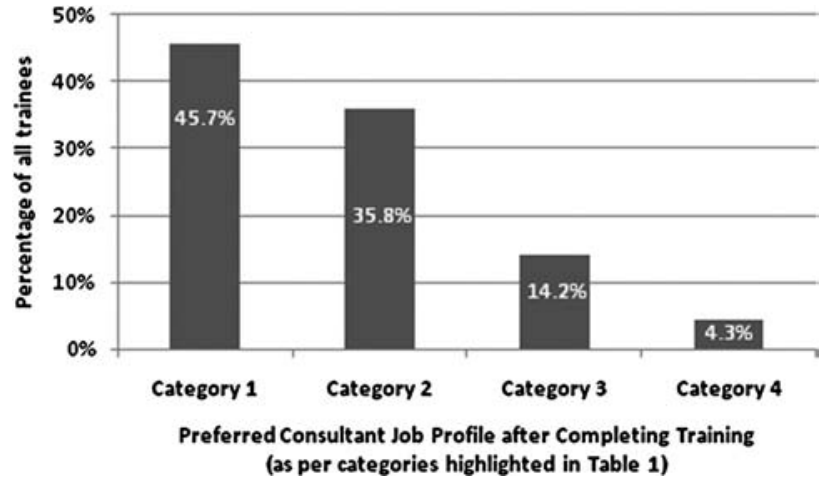

Figure 2 Proportion of trainees preferring to work in categories as defined in table 1 after completion of training.

\section{Assessing trainee 'confidence' in managing specific liver-related conditions}

A significantly lower proportion of trainees expressed a lack of confidence in managing routine and more specialised liver-related conditions if they had spent time training in a unit providing comprehensive liver services and with increasing years spent in training (table 3).

Although a similar trend towards improved confidence was seen when only trainees in their fourth year or higher were included, by which time such an exposure would have occurred for most trainees, this did not reach statistical significance owing to small numbers and the resulting type II error. Even with such specialist training, however, almost one in 10 trainees in year 4 or more did not feel confident about inserting a Sengstaken Blakemore tube, managing acute liver failure in secondary care, managing autoimmune hepatitis or referring for OLT and TIPSS; one in three was not confident about managing liver disease in the critical care setting and about half were not confident about being able to manage acute liver failure in a tertiary care setting.

\section{Discussion}

This survey suggests that the hepatology component of the unified training programme in the UK is inadequate. Although a large proportion of trainees wish to have some role in delivering liver services after training, almost one-third are dissuaded by the perceived poor quality of advanced hepatology training and its regional variability. As a result of inadequate training, a fifth of all trainees currently lack confidence in managing a range of hepatological problems.

Our survey shows that at present up to $25 \%$ of trainees in gastroenterology can complete their training to CCT without receiving clinical hepatology training in a centre delivering comprehensive liver services, with or without liver transplant experience, although training in such a unit, as is evident from this survey, significantly increases trainee confidence in managing liver-related disease. With up to $25 \%$ of new gastroenterology outpatient referrals being liver related and $50 \%$ of a general gastroenterologist's inpatient workload being 


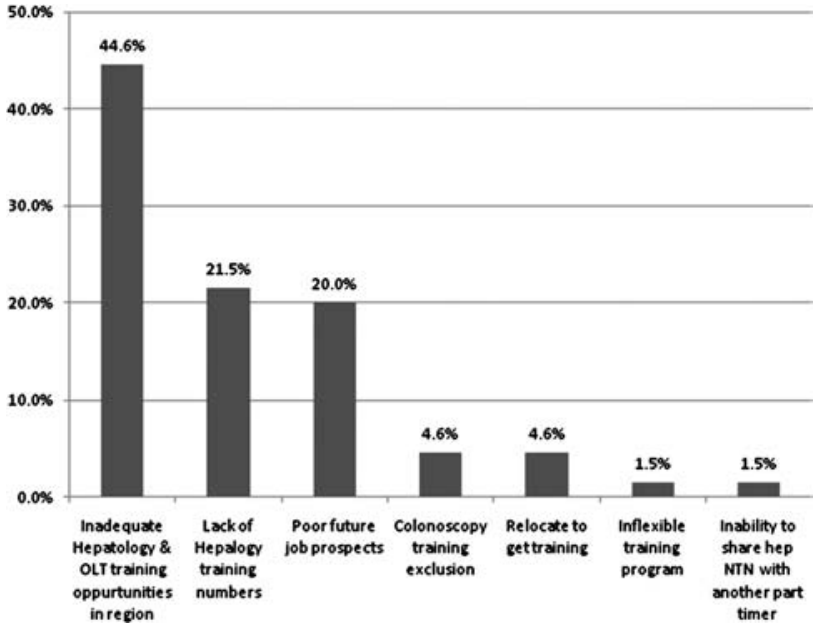

Figure 3 Reasons cited as factors dissuading trainees from pursuing their interest in hepatology as a career. NTN, national training number; OLT, orthotopic liver transplantation.

liver disease, ${ }^{5}$ this needs to be addressed. To improve the overall standard of care of patients with liver disease in the UK flexible training programmes need to be developed that can accommodate all trainees. The increasing burden of liver disease, including alcohol, non-alcoholic liver disease and the blood-borne viruses demand this. The standard of care is set in the category 3 liver centres; this should be the benchmark for all hospitals. The option of an advanced hepatology training period after basic gastroenterology training ( 2 years perhaps) for those wishing to pursue hepatology as a career is to be encouraged. This would mean creating a system for accrediting units that provide basic hepatology training after ensuring minimum standards are adhered to and where high quality of training is delivered. All trainees in gastroenterology should spend a minimum period of their training in their formative years in such units. After this period of basic training, there should be individualised training for trainees depending on their interest.

One concern raised by the trainees is lack of consultant job opportunities in hepatology. Trainees need to be confident that Trusts recognise the importance of liver disease. A balance needs to be found between the delivery of endoscopic services, both day case and out of hours, and the cost saving of reducing the readmission rates for recidivism and decompensated liver disease. Trainees should not be made to choose between training in basic colonoscopy and training in hepatology. Excluding trainees who wish to pursue hepatology from basic colonoscopy training increases the overall concern about employability in DGHs, given that these hospitals are the most in need of good hepatology services.

'Part-time' working or 'less than full-time' working needs to factored into any future workforce or manpower strategy. It is clear from this survey that $20 \%$ of the potential workforce does not intend to work full time. Assuming that all hepatology subspecialty

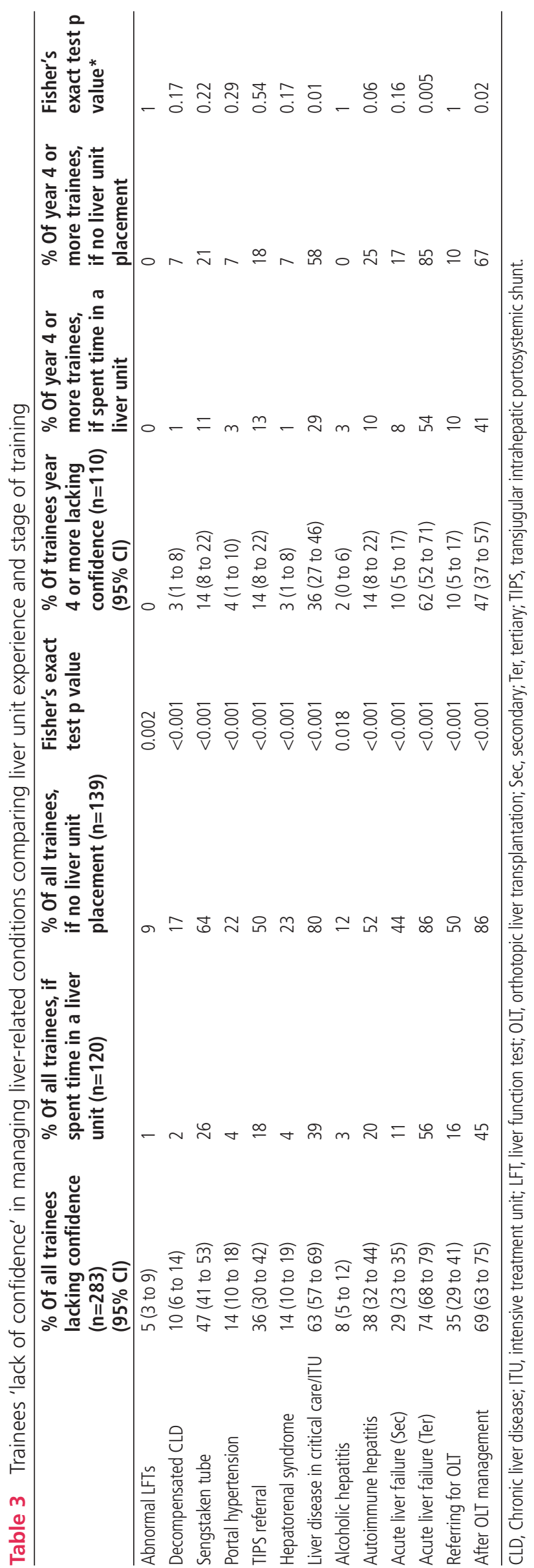


positions are filled every year and that some of these trainees will opt to work in category 3 and 4 jobs and that some will prefer part-time work, it will take more than 10 years to place a trained hepatologist in every DGH. This falls someway short of the time frame set out in the National Plan for Liver Services in 2009 for placing at least one consultant trained in advanced hepatology in every DGH. Our survey also highlights that although almost half of trainees would like to deliver liver-related services as consultants, a third are dissuaded from a career in hepatology which could further impact on this vision.

It is astonishing to note that trainees were equally confident about managing alcoholic hepatitis whether they received training in a comprehensive liver unit or not. The correct management of alcoholic hepatitis remains an area of debate among experts. We feel that the responses in our survey reflect the high burden of alcoholic liver disease that presents on the acute admission wards in the country and therefore an apparently greater 'experience' in managing this condition. Unfortunately, we did not ask about trainees' self-reported confidence in accurately diagnosing alcoholic hepatitis. We accept that this 'confidence' in managing alcoholic hepatitis does not necessarily reflect the quality of the management. However, this furthers our concerns that some patients might not be offered the level of care that should be readily available. It is also interesting that in a recent survey of gastroenterology consultants, the proportions that had no particular interest in hepatology (50\%), some interest in hepatology $(35 \%)$ and those that indentified themselves as hepatologists (15\%) mirror the proportions of trainees that would like to apply for similar profiled jobs as consultants. ${ }^{6}$

Literature suggests that doctors have a limited ability for self-assessment. ${ }^{7}$ In the absence of resources for externally assessing each of our respondents, selfassessment of trainee confidence was still accepted in this study. We realise that doing so increases the risk of social desirability bias induced in answering these questions. It may be deduced that these self-assessments reflect the effectiveness of training. The questionnaire in our study is not validated for this purpose. However, the use of self-assessments continues to be used to inform policy makers about deficiencies in postgraduate training ${ }^{8}$ and self-assessment of confidence levels developed by the Royal College of Physicians, UK continues to be used in postgraduate training portfolios. The other limitation of this survey is questionnaire bias. However, our response rates were $60 \%$, which is comparable to other surveys in which trainees or doctors were polled using questionnaires. ${ }^{4-11}$

In conclusion, this survey highlights that training in hepatology in the UK is in need of 'modernisation' if it is to deal with the increasing burden of liver disease and, in particular, if we are to fulfil the vision of the national strategy for liver service provision by 2016. A standardised curriculum implemented with the help of all stakeholders needs to be better formulated to prepare the hepatology workforce of the future.

Contributors IP and $\mathrm{MH}$ derived the study concept and design and wrote the paper. IP analysed the collected data.

Acknowledgements The authors thank Dr Adam Haycock for his help in collating the data from online responses, and Trainees in Gastroenterology for allowing the inclusion of this questionnaire in their annual survey. We also thank Dr Martin Lombard for his helpful comments.

Competing interests None.

Provenance and peer review Not commissioned; externally peer reviewed.

\section{References}

1. Thomson SJ, Westlake S, Rahman TM, et al. Chronic liver disease - an increasing problem: a study of hospital admission and mortality rates in England, 1979-2005, with particular reference to alcoholic liver disease. Alcohol Alcohol 2008;43:416-22.

2. Ashton C, Bajekal M, Raine R. Quantifying the contribution of leading causes of death to mortality decline among older people in England, 1991-2005. Health Statistics Quarterly 2010;45:100-27.

3. British Association for the Study of the Liver. The National Plan for Liver Services UK - A time to act: improving liver health and outcomes in liver disease, 2009.

4. Williams MJ, Salmon C, Austin AS, et al. Services for liver disease in district general hospitals in the UK: a national questionnaire-based survey. Clin Med 2009;9:26-9.

5. Lombard M. National Census Data 2010. England, 2011.

6. Lombard M. Gastroenterology consultant census. 2011.

7. Davis DA, Mazmanian PE, Fordis M, et al. Accuracy of physician self-assessment compared with observed measures of competence: a systematic review. JAMA 2006;296:1094-102.

8. George JT, McGrane DJ, Warriner D, et al. Protocol for a national audit on self-reported confidence levels, training requirements and current practice among trainee doctors in the UK: the Trainees Own Perception of Delivery of Care in Diabetes (TOPDOC) Study. BMC Med Educ 2010;10:54.

9. George JT, Warriner DA, Anthony J, et al. Training tomorrow's doctors in diabetes: self-reported confidence levels, practice and perceived training needs of post-graduate trainee doctors in the UK. A multi-centre survey. BMC Med Educ 2008;8:22.

10. Ramage J. Results of hepatology training questionnaire. In: Gastroenterology BSo, editor, 2002.

11. Qualia CM, Baldwin CD, Rossi TM, et al. Pediatric gastroenterology fellows, class of 2007: how well are they prepared for the future? J Pediatr Gastroenterol Nutr 2008;47:327-33. 